\title{
Radiographic Spatial Frequencies Essential to the Diagnosis of Incipient Interproximal Lesions
}

\author{
R. L. WEBBER and P. H. KOZIOL
}

National Institutes of Health, Bethesda, Maryland 20014, USA, and

1126 South 7th St, Ann Arbor, Michigan 48103

The effects of selective obscuration of dental radiographs containing known incipient interproximal lesions suggest that the amount of resolution required for reliable diagnosis is anisotropic and can be related to the spatial frequency characteristics of the image. Frequencies less than the limiting resolution of existing screen-film techniques seem to be particularly important.

Factors that influence diagnostic performance associated with the use of dental radiographs have been shown to be relatively task specific. ${ }^{1}$ Radiographic features essential to one diagnostic task may obscure information required for another; hence, optimization requires specification of the diagnostic task in terms that can be objectively related to other elements in the radiographic chain.

The goal of this investigation is to determine position invariant image characteristics that are essential to the reliable performance of a specific diagnostic task, that is, the detection of incipient interproximal lesions from bitewing-type radiographs.

The approach involves the introduction of frequency-specific "noise" into the system and noting the effects on diagnostic accuracy obtainable from radiographs containing interproximal lesions of known size and location. Interpretation of the resulting data is facilitated by considering pertinent elements of this diagnostic system as being linear. This means that performance characteristics of the various components are assumed to be constant, with the output of the system being proportional to the input. Fortunately, this

Presented at the general session of the AADR, April 1975, New York, NY.

Received for publication July 14, 1975.

Accepted for publication March 12, 1976. assumption can be justified for nearly all elements involved in the production of a typical radiograph.2

RATIONALE.-The source of a radiographic image may be defined as a multidimensional distribution of X-ray photons that contains diagnostically meaningful information. The effects of this distribution can be expressed in a variety of ways that are influenced by the transfer characteristics of the radiographic system. Since the image-forming components are relatively linear, one can predict the overall effect that the system will have on any image by analyzing its effect on the image of a single point. This measured effect on image intensity is called the "point spread function" of the system. It completely specifies the nature and extent of the image degradation irrespective of the type of pattern being registered.

The point spread function, $F(x, y)$, can be alternatively expressed as the modulation transfer function of the system, $f(\theta, p)$, since the two are related by the following equations that are two-dimensional Fourier transforms of each other ${ }^{3}$ :

$$
\begin{gathered}
f(\theta, p)=\iint_{-\infty}^{\infty} \mathrm{F}(x, y) e^{-2 \pi i(x \theta+y \rho) d x d y} \\
\mathrm{~F}(x, y)=\int_{-\infty}^{\infty} f(\theta, \rho) e^{i\left(x \theta+y_{\rho}\right) d \theta d \rho}
\end{gathered}
$$

These equations, respectively, express image degradation resulting from linear elements in terms of the effect they have on all spatial frequencies that may pass through the system and vice versa. Image degradation can be unequivocally related to an intensity-modulated spectrum of spatial frequencies as shown in Figure 1.

The question of particular interest in this investigation is whether certain of these 


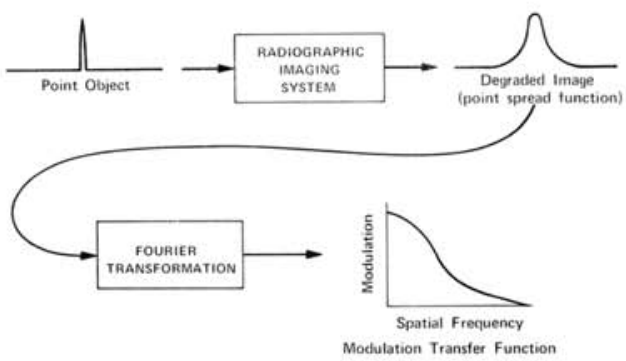

Fig 1.-Modulation transfer function: Fourier transform of intensity distribution produced by system when input gray level pattern is single point.

spatial frequencies are more important than others when a specific diagnostic task is performed. Since many factors, such as exposure geometry, focal spot configuration, photon energy distribution, film characteristics, processing conditions, and others determine the modulation transfer function of the system, it is important to know which frequencies are essential for diagnostic purposes. Such information would be most helpful in selecting components with a specific task in mind. It would also provide an objective basis for specifying the minimum transfer characteristics consistent with a criterion level of diagnostic performance for any given application.

\section{Materials and Methods}

One way to determine which spatial frequencies are essential to a diagnostic task is to selectively obscure information at each frequency and note the effects on diagnostic performance. This can be done in a variety of ways, but perhaps the simplest approach involves masking standardized radiographs through the addition of bands that have sinusoidal variations in intensity at various spatial frequencies, as seen in Figure 2 for one particular frequency. Although suitably randomized undimensional obscuration of this sort should be done in all directions to unambiguously generalize results in the frequency domain, anisotropic effects can be demonstrated by making a single set of orthogonal measurements. Sampling in two directions also provides a simple means for demonstrating differences in spatial frequency characteristics with a reasonable number of measurements. Hence, this ap-

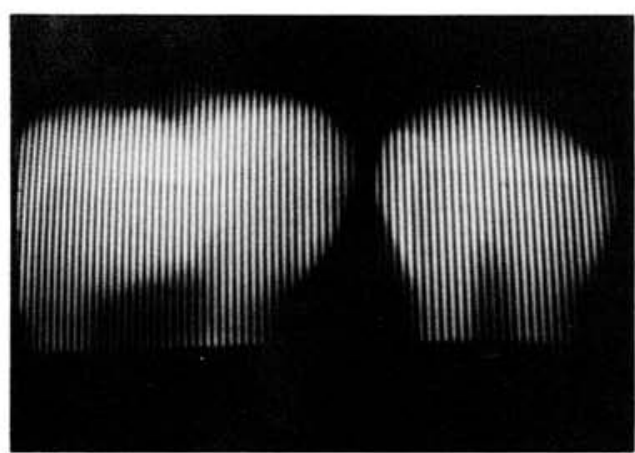

Fig 2.-Sinusoidally varying bands added to television display of portion of bitewing-type radiograph. Spatial frequency of bands is defined as number of complete oscillations (dark-light pairs) per unit length.

proach is considered a reasonable basis for a preliminary investigation of the task-dependent nature of image quality.

Composite displays of this sort were produced by means of a modified closed circuit television system of the type schematized in Figure 3. A high-quality vidicon that has a 25-mm diameter faceplate was optically coupled to specially prepared dental radiographs with the use of a magnification factor of approximately one half. Since a vidicon of this sort is easily capable of passing frequencies less than 20 cycles $/ \mathrm{mm}, 2$ this represents a band pass of at least 10 cycles/ $\mathrm{mm}$ referred back to the plane of the film. This was considered adequate since the masking frequencies used to test performance never exceeded 3 cycles $/ \mathrm{mm}$ when comparably scaled.

An accurate measure of diagnostic performance was made possible by using special

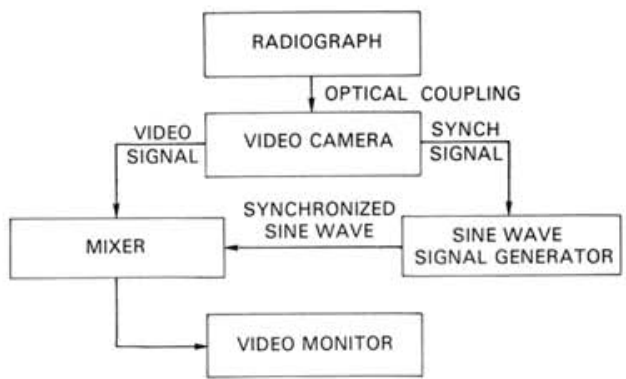

FIG 3.-Schematic diagram of closed circuit television system used to mask radiographic display with sinusoidally varying bands. 


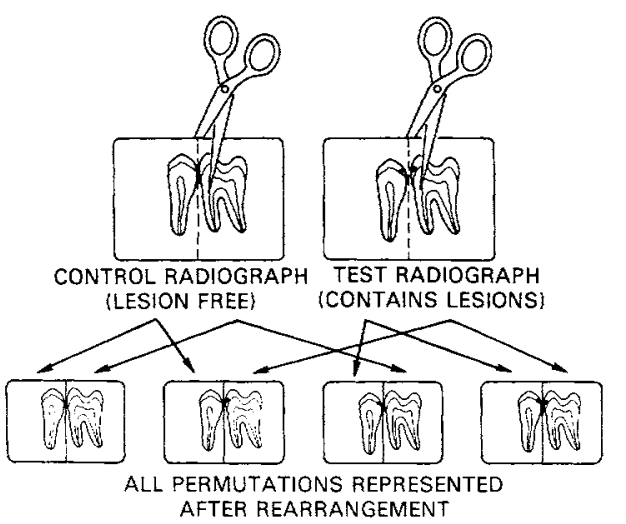

Fig 4.-Technique for balancing number of induced lesions in radiographs used for psychophysical analysis.

radiographs that were identical in every respect except for the fact that some contained evidence of one or more induced interproximal lesions. To assure that only the presence or absence of a lesion was the basis for diagnosis, several radiographs of suitably mounted, extracted teeth (a mandibular first molar and a mandibular second premolar) were made before the induction of $1-\mathrm{mm}^{3}$ hemispherical lesions in the respective areas of proximal contact.

A second series of radiographs was then produced using identical geometry after the induced lesions had been filled with modeling clay to simulate the radiodensity associated with carious enamel. The effects of soft tissue scatter were simulated with an 11-mm thick slab of tissue equivalent material (Mix D) which was placed in the primary beam during the exposure of all films. In other respects, the technique was conventional, involving long cone geometry consistent with a 1.1-second exposure at a 65 kilovolt peak and 10 ma on Kodak Ultraspeed film ${ }^{\star}$ from a Ritter X-ray machine ${ }^{b}$ that has filtration equivalent to $2.5 \mathrm{~mm}$ of A1. Processing was in accord with manufacturers' instructions, using fresh Kodak liquid X-ray developer. ${ }^{\mathrm{a}}$ This resulted in radiographs that were conventional both in appearance (mean density of proximal enamel 0.7 ) and in terms of the amount of radiation required to produce them $(0.2$ roentgen measured at the tip of the cone).

* Eastman-Kodak, Rochester, NY.

b Ritter Dental Co., Rochester, NY.

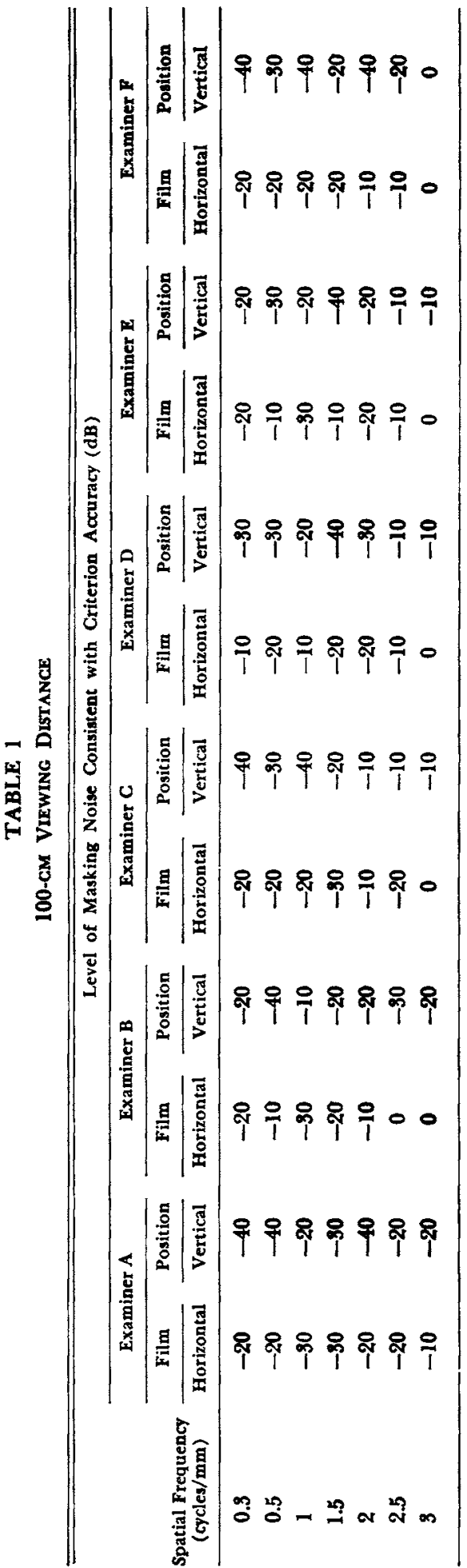


After fixing and drying, these radiographs were carefully cut in a way that permitted individual teeth to be rearranged as shown in Figure 4. Examples of each presentation were carefully checked for the presence of any artifacts that might be specific to a particular arrangement. Of those considered to be free of such artifacts, one or more of each type were ultimately selected as source radiographs to be evaluated by a total of six clinically experienced observers. Each observer participated in the study on a blind basis by assessing randomly selected radiographs projected on a $0.30-\mathrm{cm}$ (12-inch) video monitor at a distance of $100 \mathrm{~cm}$. Masking bands having sinusoidal variations in intensity were generated as shown in Figure 3 and additively mixed with the video display of each radiograph as seen in Figure 2. Each examiner was apprised of the fact that any particular interproximal area to be evaluated had a 0.5 chance of containing an induced radiographic lesion. The actual area of concern (either molar or premolar proximal enamel) was randomly selected for each trial at the time of the experiment. Each participant attempted to determine the presence or absence of a lesion while the modulation of the masking sinusoidal bands and spatial frequency were systematically altered between trials. At each of seven selected frequencies, modulation was thus decreased by $10 \mathrm{~dB}$ whenever a diagnostic error was made until five consecutive correct responses could be registered. Since the a priori probability of a lesion was 0.5 , criterion accuracy could be expected to occur by chance with a probability of $(0.5)^{5}$, that is, less than $4 \%$ of the time. Thus, each examiner attempted to detect lesions in randomly selected areas in a series of independent trials that were characterized by a serially decreasing intensity of superimposed sinusoidal bands until criterion accuracy could be demonstrated at each of seven spatial frequencies. The entire process was then repeated with the radiographs rotated $90^{\circ}$ relative to the video camera so that the superimposed sinusoidal bands were now displayed perpendicular to the long axes of the teeth. In this way, it was possible to test whether the effects of frequency-dependent noise were anisotropic insofar as diagnosis of interproximal lesions was concerned.

To test whether the effects of the bands were relatively independent of viewing ge- ometry, three of the examiners repeated the experiment with the bands parallel to the long axes of the teeth at a distance of $25 \mathrm{~cm}$ from the television screen.

The video system was adjusted in such a way that the average luminance of a radiographic image including masking bands was approximately $15 \mathrm{~cd} /$ meter $^{2}$. Since this value lies in the lower photopic range, its effect on the visual acuity of the examiners was considered inconsequential. 5 The intensity added by the bands per se ranged downward from a maximum of $35 \mathrm{~cd} /$ meter $^{2}$ as the modulation was reduced in decrements of $10 \mathrm{~dB}$ until criterion accuracy was achieved. This peak brightness was an order of magnitude less than that associated with saturation of the video system, so that the brightness changes were functionally related to the modulation levels of the sine wave generator. This made it possible for the modulation to be considered as a meaningful measure of the level of masking associated with any particular display.

No attempt was made to control the phase of the masking bands relative to the interproximal areas of interest, so that for all practical purposes, the phase relationship within any given display could be considered random.

\section{Results}

Pertinent data are given in Table 1 which presents the relative modulation of the masking that could be tolerated while achieving criterion accuracy by each participant at each spatial frequency when the viewing distance was $100 \mathrm{~cm}$. Results are compiled for bands registered both parallel and perpendicular to the long axes of the teeth, respectively, as noted by the headings identi-

TABLE 2

Group Performance

\begin{tabular}{lcc}
\hline \hline $\begin{array}{c}\text { Spatial } \\
\begin{array}{c}\text { Frequency } \\
\text { (cycles/ } \\
\text { mm) }\end{array}\end{array}$ & $\begin{array}{c}\text { Horizontal } \\
\text { Average/SD }\end{array}$ & $\begin{array}{c}\text { Vertical } \\
\text { Average/SD }\end{array}$ \\
\hline 0.3 & $-18.3 / 4.1$ & $-31.7 / 9.8$ \\
0.5 & $-16.7 / 5.2$ & $-33.3 / 5.2$ \\
1 & $-23.3 / 8.2$ & $-25.0 / 12.2$ \\
1.5 & $-21.7 / 7.5$ & $-28.3 / 9.8$ \\
2 & $-15.0 / 5.5$ & $-26.7 / 12.1$ \\
2.5 & $-11.7 / 7.5$ & $-16.7 / 8.2$ \\
3 & $-1.7 / 4.1$ & $-11.7 / 7.5$ \\
\hline
\end{tabular}




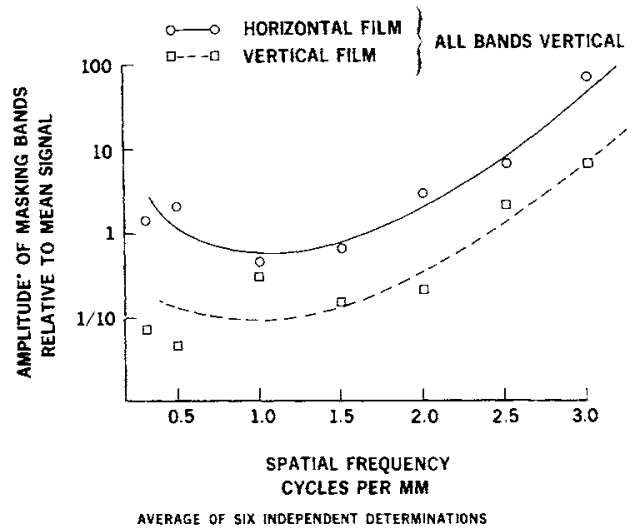

Fig 5.-Relative modulation of masking bands that could be tolerated while performing at criterion accuracy as function of spatial frequency when viewing distance was $100 \mathrm{~cm}$. Average of six independent determinations.

fying the film position relative to the video system.

Performance trends are appreciated more easily by averaging findings at each frequency (Table 2). These results are plotted in Figure 5 where the modulation levels are expressed as equivalent amplitudes relative to the mean video signal produced by the radiographs. The two curves were fitted by eye and corresponded to the averaged results in the two spatial dimensions (horizontal and vertical) for which the effects of the masking bands were determined.

The data that resulted from reducing the viewing distance to $25 \mathrm{~cm}$ are given in Table 3. Since the variation associated with random phase differences would necessarily depend on the spatial frequency involved, homogeneity of variance could not be assumed. Hence, statistical tests were appropriately limited to nonparametric methods wherein the data were dichotomized at each spatial frequency according to whether the observed values were above or below the median. ${ }^{6}$ In each instance, the hypothesis that the distribution of observed values was distributed independent of spatial frequency could be rejected via a chi-square test with a level of confidence greater than 0.95 . Indeed, all values less than the median of -20 $\mathrm{dB}$ were confined to spatial frequencies lower than 3 cycles $/ \mathrm{mm}$, suggesting that the lower frequencies are relatively more critical to accurate diagnosis irrespective of orientation.

When the data were similarly evaluated for the effect of orientation on the masking bands relative to the teeth, the anisotropic effects were likewise found to be statistically significant, in this instance the level of confidence exceeding 0.999 .

The lack of data for the $25-\mathrm{cm}$ viewing distance precluded meaningful statistical evaluation, but the results were in keeping with what would be expected under the circumstances.

\section{Discussion}

The high degree of dispersion in the data as evidenced by the standard deviation noted in Table 2 is not surprising since these results include all learning errors of the participants. As previously mentioned, the phase of the masking bands relative to the interproximal areas of interest was randomly determined from one trial to the next, which also resulted in relatively large deviations in performance.

As a result of this latter condition, one diagnostic area might have an entirely different appearance than another depending on the chance location of the bands. This effect is particularly noticeable at low frequencies where the bands are necessarily

TABLE 3

25-CM VIEWING DISTANCE

\begin{tabular}{lcccc}
\hline \multirow{2}{*}{$\begin{array}{c}\text { Spatial } \\
\text { Frequency } \\
\text { (cycles/mm) }\end{array}$} & \multicolumn{5}{c}{$\begin{array}{c}\text { Level of Masking Noise Consistent with } \\
\text { Criterion Accuracy (dB) }\end{array}$} \\
\cline { 2 - 5 } & Examiner A & Examiner D & Examiner E & Average \\
\hline 0.3 & -10 & -20 & -20 & -16.7 \\
0.5 & -20 & -20 & -40 & -26.7 \\
1 & -20 & -30 & -30 & -26.7 \\
1.5 & -30 & -40 & -30 & -33.3 \\
2 & -20 & -40 & -20 & -26.7 \\
2.5 & -20 & -30 & -20 & -23.3 \\
3 & -20 & -30 & -20 & -23.3 \\
\hline
\end{tabular}




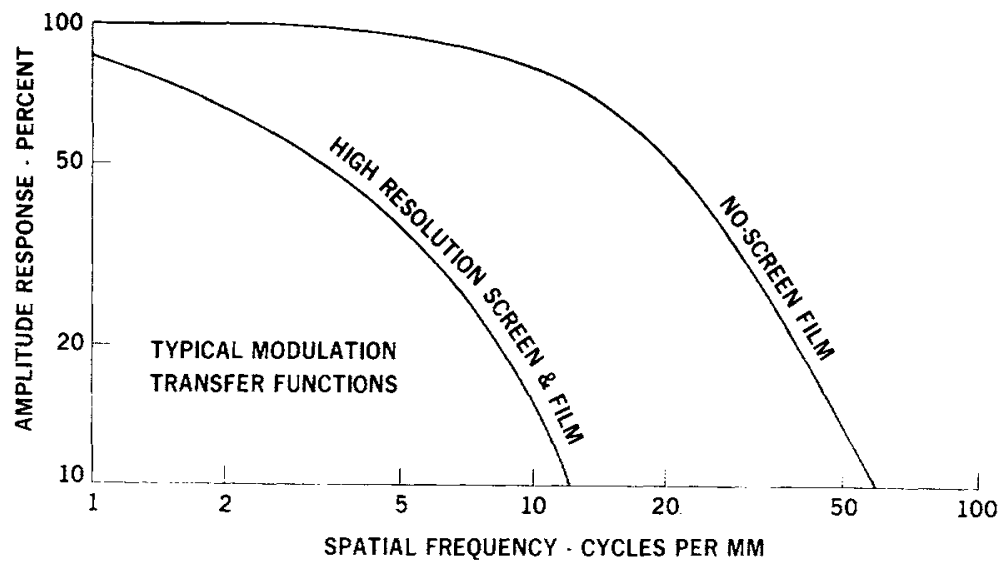

FIG 6.-Typical modulation transfer functions of screen and no-screen radiographic detection systems.

wide. It is for this reason that the data were analyzed nonparametrically.

It is interesting to note that spatial frequencies less than 3 cycles $/ \mathrm{mm}$, which were shown to be more important for diagnostic accuracy, lie well below the limiting resolution of the film-screen combination shown in Figure 6. If this observation can be substantiated and generalized by more comprehensive means, it may be possible to use higher-speed detectors that could reduce exposure to radiation by a factor of 20 or more with little if any sacrifice in diagnostic accuracy. In this regard, it is recognized that modulating an image with fixed pattern noise is not equivalent to spatial frequency filtering. Hence, any conclusions based on psychophysical experiments of this sort must be confirmed by more direct methods. This approach does, however, provide a tractable basis for generating frequency-specific information that can be verified in a variety of ways.

For example, the pronounced effect of the orientation of the masking bands relative to the long axes of the teeth can be generalized and confirmed by a simple demonstration involving anisotropic distribution of high spatial frequencies. This is easily accomplished by astigmatically blurring a radiograph containing evidence of an interproximal defect, since defocusing is a convenient means for low-pass filtering of spatial frequencies.

For the purpose of this demonstration, astigmatic blurring was accomplished by interposing a cylindrical lens in the light path between the radiograph and the television camera. As a consequence, vertical lines were inappropriately focused when horizontal lines were seen sharply and vice versa. Figure 7, top, shows the lesion with no astigmatic distortion; Figure 7 , center, shows what happens to the image when it is blurred in the horizontal direction. Notice that vertical lines are obscured, but the defect is still easily detected. Figure 7 , bottom is the result of vertical blurring wherein horizontal lines are obscured. In this instance, the lesion all but disappears as might be suspected from the more pronounced effect of modulation changes manifest as horizontal bands at right angles to the long axes of the teeth. Preliminary findings from experiments based on motion-degraded images likewise confirm that high spatial frequencies are not essential for the reliable detection of interproximal lesions as suggested by these data.

Regarding the data produced at a $25-\mathrm{cm}$ viewing distance, it is recognized that visual performance is influenced by the angle that a given image subtends at the eye. In general, it is maximized for spatial frequencies approaching 1 cycle per minute of arc. Hence, at $25 \mathrm{~cm}$ one would expect performance to be effectively shifted toward lower spatial frequencies. The fact that at 3 cycles/ mm criterion accuracy could not be accomplished by any of the participants viewing the screen at $25 \mathrm{~cm}$ with modulations greater than $20 \mathrm{~dB}$ would tend to confirm this sup- 

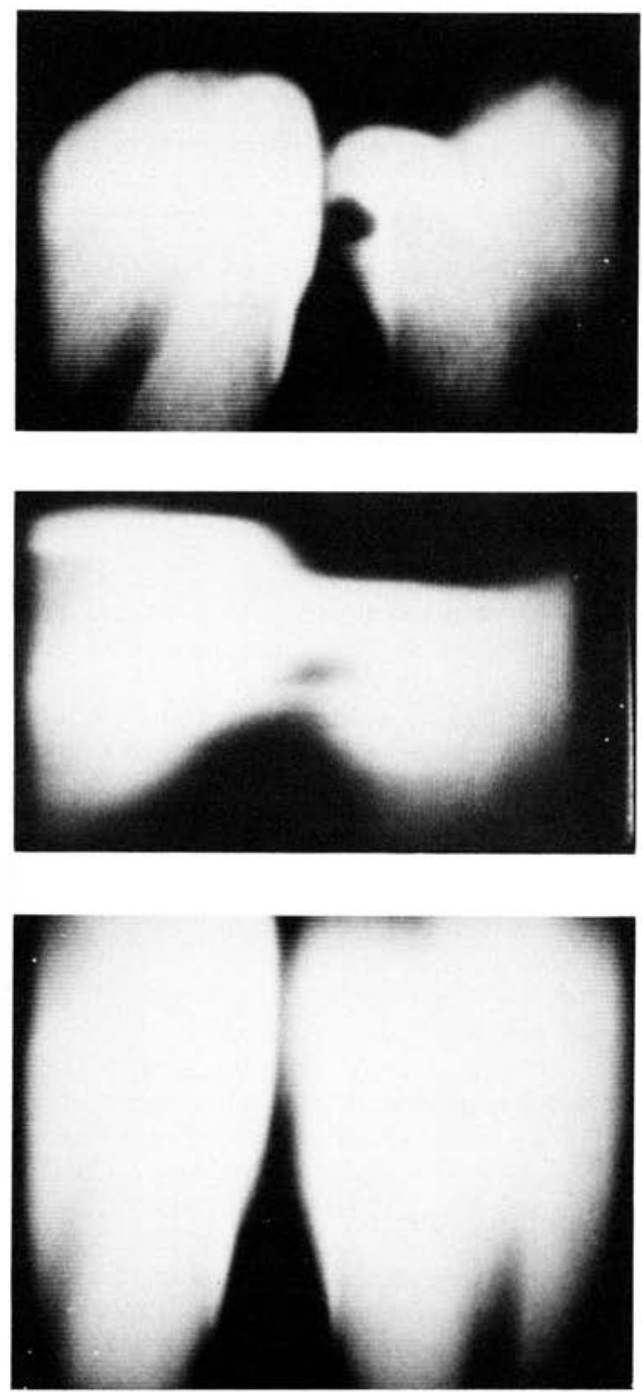

FIG 7.-Reproductions of slides showing, respectively, effects of astigmatic blurring. Top, no blur; center, horizontal blur; and bottom, vertical blur.

position. In any case, one cannot account for the relative diagnostic significance of spatial frequencies less than 3 cycles $/ \mathrm{mm}$ (referred back to the plane of the film) on this basis since the eye is actually more sensitive to higher frequencies as produced at a $100-\mathrm{cm}$ viewing distance as in this experiment.

\section{Conclusions}

The effects of fixed pattern noise on the interpretation of dental radiographs containing known lesions suggest that reliable detection of incipient interproximal caries is influenced by the spatial frequency of information visibly displayed. Frequencies less than the limiting resolution of existing screen-film radiographic techniques were observed to be particularly important. The data also permit demonstration that significantly less resolution is required horizontally than vertically to achieve a criterion level of diagnostic accuracy. These findings are consistent with the notion that accurate diagnosis may be possible with less resolution than is currently produced by conventional bitewing radiographic techniques. If this is true and the result can be generalized in two dimensions, more convenient means for radiographically detecting caries can be developed that require less exposure of the patient to ionizing radiation.

The authors acknowledge the dentists and associated staff members of the clinical dental services section, National Institute of Dental Research who participated in the psychophysical determinations as diagnostic interpreters, and the efforts of Drs. John Folio and William Wright who helped to make this project possible.

\section{References}

1. Webrer, R.L., and StARK, L.: Influence of Fogging Radiation and Mode of Display on the Interpretation of Dental Caries from Conventional Radiographs, Invest Radiol 7: 506516, 1972.

2. GREgG, E.C.: Assessment of Radiographic Imaging, Am J Roentgenol 97: 776-792, 1966.

3. Jennison, R.C.: Fourier Transforms and Convolutions for the Experimentalist, New York: Pergamon Press, 1961.

4. Webber, R.L.; Benton, P.A.; and Ryge, G.: Diagnostic Variations in Radiographs, Oral Surg 26: 808-809, 1968.

5. Graham, C.H.; Bartlett, N.R.; Brown, J.L.; Hsia, Y.; Mueller, C.G.; and Riggs, L.A.: Vision and Visual Perception, New York: John Wiley \& Sons, 1965.

6. Siegel, S.: Non Parametric Statistics, New York: McGraw-Hill, 1956. 